\title{
Increasing selections from increasing multifunctions
}

\author{
Nikolai S. Kukushkin*
}

April 14, 2012

\begin{abstract}
We study when the existence of an increasing selection can be derived from the monotonicity of a multifunction w.r.t. an extension of the basic order from points to subsets. The most interesting results emerge when the target is a lattice and monotonicity is interpreted in one of the ways suggested by A.F. Veinott, Jr. An increasing selection exists if (1) the multifunction is weakly ascending while every value satisfies a completeness condition, e.g., is chain-complete, or (2) the multifunction is ascending while the target is a sublattice of the Cartesian product of a finite number of chains.

Key words: increasing selection; ascending multifunction; weakly ascending multifunction
\end{abstract}

\footnotetext{
${ }^{*}$ Russian Academy of Sciences, Dorodnicyn Computing Center, 40, Vavilova, Moscow 119333 Russia E-mail: ququns@inbox.ru
} 


\section{Introduction}

We denote $\mathfrak{B}_{X}$ the set of all nonempty subsets of a given set $X$. A mapping $T \rightarrow \mathfrak{B}_{X}$ is called a multifunction (from $T$ to $X$ ). A selection from a multifunction $F: T \rightarrow \mathfrak{B}_{X}$ is a mapping $f: T \rightarrow X$ such that $f(t) \in F(t)$ for every $t \in T$.

Given a poset $X$, a binary relation $\geq^{*}$ on $\mathfrak{B}_{X}$ is called an extension of the basic order (from points to subsets) if

$$
\{y\} \geq^{*}\{x\} \Longleftrightarrow y \geq x
$$

for all $y, x \in X$. Let $T$ be a poset too. A multifunction $F: T \rightarrow \mathfrak{B}_{X}$ is increasing w.r.t. $\geq^{*}$ if $F\left(t^{\prime}\right) \geq^{*} F(t)$ whenever $t^{\prime}>t$. Note the strict inequality in the last condition; it is natural when dealing with binary relations that need not be reflexive.

This paper strives to derive the existence of increasing selections from the monotonicity of a multifunction w.r.t. various (natural) extensions of the basic order. Besides monotonicity, we allow "point-wise" restrictions on the multifunction, usually, some sort of the completeness of values. However, we never impose more complicated restrictions as was typical in the pioneering works on the subject [17, 3]. We also briefly address the possibility to extend a "partial" increasing selection to the whole domain, especially, the existence of an increasing selection with a given value at a given point.

The most interesting results emerge when $X$ is a lattice rather than just a poset and the basic order is extended to $\mathfrak{B}_{X}$ in one of the ways suggested by Veinott 20 . Funnily, none of the extensions need be reflexive, and only one of them is transitive. On the other hand, there is an extension of the order to $\mathfrak{B}_{X}$, which coincides with one of Veinott's when $X$ is a chain, is a partial order for any poset $X$, and ensures the existence of an increasing selection from every increasing multifunction without any further assumptions.

An interest in the existence of increasing selections can be justified, e.g., by the fact that most fundamental fixed point theorems based on monotonicity [18, 1, 11] relate to single-valued functions. In game theory, the existence of a Nash equilibrium in a game with "strategic complementarities" is usually obtained by applying Tarski's fixed point theorem to increasing selections from the best response correspondences [19, 12, 21], even though there are fixed point theorems in the literature that can work in the absence of increasing selections, see, e.g., [17, Theorem 1.1] or [16, Lemma 2.8]. There is also a growing literature on aggregative games [14, 5, 6, 7, 8, 2, 4], where the existence of an equilibrium can be derived from a wider range of monotonicity conditions (e.g., the best responses may be increasing in some variables and decreasing in others). Every result of this kind needs monotone selections.

A poset $X$ is called chain-complete upwards/downwards if $\sup Y / \inf Y$ exists in $X$ for every chain $Y \in \mathfrak{B}_{X}$; in this case, $Y \in \mathfrak{B}_{X}$ is chain-subcomplete upwards/downwards if $\sup Z \in Y /$ $\inf Z \in Y$ for every chain $Z \in \mathfrak{B}_{Y} \subseteq \mathfrak{B}_{X}$. A poset $X$ is called chain-complete if it is chain-complete both upwards and downwards; $Y \in \mathfrak{B}_{X}$ is then chain-subcomplete if it is chain-subcomplete both upwards and downwards.

Given a poset $X$ and $Y \in \mathfrak{B}_{X}$, we introduce a notation for the subsets of maximal $/ \mathrm{minimal}$ 
elements of $Y$, denoting

$$
Y^{+}:=\{x \in Y \mid \nexists y \in Y[y>x]\} ; \quad Y^{-}:=\{x \in Y \mid \nexists y \in Y[y<x]\} .
$$

A subset $Y \in \mathfrak{B}_{X}$ is called supported below/above if $Y^{-} \neq \emptyset / Y^{+} \neq \emptyset$. By Zorn's Lemma, if a poset is chain-complete downwards/upwards, then it is supported below/above.

\section{Lattices as targets}

Veinott [20] defined four extensions of the order from a lattice $X$ to $\mathfrak{B}_{X}\left(Y, Z \in \mathfrak{B}_{X}\right)$ :

$$
\begin{gathered}
Y \geq^{\wedge} Z \rightleftharpoons \forall y \in Y \forall z \in Z[y \wedge z \in Z] ; \\
Y \geq^{\vee} Z \rightleftharpoons \forall y \in Y \forall z \in Z[y \vee z \in Y] ; \\
Y \geq^{\mathrm{Vt}} Z \rightleftharpoons\left[Y \geq^{\wedge} Z \& Y \geq^{\vee} Z\right] ; \\
Y \geq^{\mathrm{wV}} Z \rightleftharpoons \forall y \in Y \forall z \in Z[y \vee z \in Y \text { or } y \wedge z \in Z] .
\end{gathered}
$$

The relation $\geq^{\mathrm{Vt}}$ is antisymmetric and transitive on $\mathfrak{B}_{X}$; it is reflexive on sublattices. Neither $\geq^{\mathrm{VV}}$, nor $\geq^{\wedge}$ or $\geq^{\vee}$ need even be transitive. Multifunctions increasing w.r.t. $\geq^{\mathrm{Vt}}\left(\geq^{\mathrm{wV}}\right)$ were called (weakly) ascending in [20].

Proposition 2.1. Let $X$ be a lattice, $T$ be a poset, $F: T \rightarrow \mathfrak{B}_{X}$ be increasing w.r.t. $\geq \wedge$, and every $F(t)(t \in T)$ be supported below. Then there exists an increasing selection from $F$.

Proof. Clearly, any selection from $F^{-}$is increasing (here and further on, $F^{-}(t)$ is the set of minimal elements of $F(t)$ ).

Remark. Proposition 2.1 remains meaningful and valid when $X$ is a semilattice. Naturally, the dual version is valid as well.

Theorem 1. Let $X$ be a lattice, $T$ be a poset, $F: T \rightarrow \mathfrak{B}_{X}$ be increasing w.r.t. $\geq^{\mathrm{wV}}$, and every $F(t)(t \in T)$ be chain-complete upwards and supported below. Then there exists an increasing selection from $F$.

The proof is deferred to Section 4.1. Again, the dual version is valid as well.

Remark. Theorem 3.2 of 20 established the existence of an increasing selection from a weakly ascending multifunction under more stringent assumptions: $X$ was a complete lattice and every $F(t)$ was chain-subcomplete in $X$. Unfortunately, that result seems to have never been published at all.

The upward chain-completeness of every $F(t)$ in Theorem 1 cannot be replaced with just being supported above. The existence of an increasing selection with a given value at a given point also cannot be asserted. 
Example 2.2. Let $X:=[-1,1]$ and $F: X \rightarrow \mathfrak{B}_{X}$ be this: $F(0):=X \backslash\{0\} ; F(x):=\{x / 2\}$ for $x \in X \backslash\{0\}$. Clearly, every $F(x)$ is supported both above and below, whereas $F$ is increasing w.r.t. $\geq^{\mathrm{wV}}$; however, $F$ admits no increasing selection, nor fixed point for that matter.

Example 2.3. Let $X:=\{0,1\}$ and $F: X \rightarrow \mathfrak{B}_{X}$ be this: $F(0):=X ; F(1):=\{0\}$. Clearly, $F$ is increasing w.r.t. $\geq^{\mathrm{wV}}$ (even $\left.\geq^{\wedge}\right)$; however, $F$ admits no increasing selection such that $f(0)=1$.

Naturally, Theorem 1 applies when $T$ is an arbitrary poset and every $F(t)$ is finite. Alternatively, we can drop all restrictions on values $F(t)$ and assume that $T$ is finite instead.

Proposition 2.4. Let $X$ be a lattice, $T$ be a finite poset, and $F: T \rightarrow \mathfrak{B}_{X}$ be increasing w.r.t. $\geq^{\mathrm{wV}}$. Then there exists an increasing selection from $F$.

The proof is deferred to Section 4.2 .

Remark. The validity of Proposition 2.4 was mentioned in [20], but a different proof was suggested.

The finiteness of $T$ in Proposition 2.4 cannot be replaced with, say, chain-completeness.

Example 2.5. Let $X:=[0,1] \subset \mathbb{R}, T:=\{(0,0)\} \cup\left\{\left(t_{1}, t_{2}\right) \in \mathbb{R}^{2} \mid t_{1}, t_{2}>0 \& t_{1}+t_{2}=1\right\} \subset \mathbb{R}^{2}$, and $F: T \rightarrow \mathfrak{B}_{X}$ be defined as follows: $\left.\left.F(0,0):=\right] 0,1\right]$ and $F\left(t_{1}, t_{2}\right):=\left\{t_{1}\right\}$ when $t_{1}>0$. Thus, $F$ is increasing w.r.t. $\geq^{\mathrm{wV}}, T$ is a poset without infinite chains (hence chain-complete), $X$ is a complete chain, and every $F(t)$ is chain-subcomplete upwards. However, there is no increasing selection.

When it comes to monotonicity w.r.t. $\geq^{\mathrm{Vt}}$, we can drop all restrictions on $T$ and $F(t)$, and only impose a peculiar restriction on $X$.

Theorem 2. Let $X$ be a sublattice of the Cartesian product of a finite number of chains. Let $T$ be a poset and $F: T \rightarrow \mathfrak{B}_{X}$ be increasing w.r.t. $\geq^{\mathrm{Vt}}$. Let $t^{0} \in T$ and $x^{0} \in F\left(t^{0}\right)$. Then there exists an increasing selection $f$ from $F$ such that $f\left(t^{0}\right)=x^{0}$.

The proof is deferred to Section 4.3 .

\section{General posets as targets}

If $X$ is a poset, but not a lattice, relations (2) need not make any sense. However, some analogs can be defined (again, $Y, Z \in \mathfrak{B}_{X}$ ):

$$
\begin{gathered}
Y \geqslant^{\mathrm{Up}} Z \rightleftharpoons \forall y \in Y \backslash Z \forall z \in Z[y>z] ; \\
Y \geqslant^{\mathrm{Dn}} Z \rightleftharpoons \forall y \in Y \forall z \in Z \backslash Y[y>z] ; \\
Y \geqslant Z \rightleftharpoons\left[Y \geqslant \text { Up } Z \& Y \geqslant{ }^{\operatorname{Dn}} Z\right] ; \\
Y \geqslant^{\mathrm{w}} Z \rightleftharpoons \forall y \in Y \backslash Z \forall z \in Z \backslash Y[y>z] .
\end{gathered}
$$

All relations (3) are reflexive; only (3c) is also transitive and anti-symmetric, i.e., a partial order. 
Proposition 3.1. If both $Y, Z \in \mathfrak{B}_{X}$ are chains, then each relation (3) implies the corresponding relation (2). If $Y \cup Z$ is a chain, then each relation (2) implies the corresponding relation (3).

A straightforward proof is omitted.

To my knowledge, relations (3) are not popular in the literature. LiCalzi and Veinott [10] considered the conjunction of $(2 \mathrm{c})$ with the requirement that $y$ and $z$ are comparable in the basic order whenever $y \in Y$ and $z \in Z$; this relation is equivalent to the conjunction of (3c) with the same requirement. Much more popular are these extensions of the basic order to $\mathfrak{B}_{X}$ :

$$
\begin{gathered}
Y \geq^{\inf } Z \rightleftharpoons \forall y \in Y \exists z \in Z[y \geq z] ; \\
Y \geq^{\text {sup }} Z \rightleftharpoons \forall z \in Z \exists y \in Y[y \geq z] ; \\
Y \geq Z \rightleftharpoons\left[Y \geq^{\text {sup }} Z \& Y \geq^{\text {inf }} Z\right] . \\
Y \gg Z \rightleftharpoons \forall y \in Y \forall z \in Z[y \geq z] .
\end{gathered}
$$

All of them are transitive; all relations (4) are also reflexive.

Proposition 3.2. For any $Y, Z \in \mathfrak{B}_{X}$, each of the first three relations (2), as well as each of the first three relations (3), implies the corresponding relation (4). The relation (5) implies all relations (2), (3), and (4).

A straightforward proof is omitted.

If $T$ is a poset, then $F: T \rightarrow \mathfrak{B}_{X}$ is increasing w.r.t. $\gg$ if and only if every selection from $F$ is increasing. Assuming the Axiom of Choice, we obtain the existence of an increasing selection, as well as the possibility to extend any partial selection to the whole $T$.

Proposition 3.3 ([17, Theorem 1.7]). Let $T$ and $X$ be posets; let $F: T \rightarrow \mathfrak{B}_{X}$ be increasing w.r.t. $\geq^{\text {inf; }}$ let every $F(t)$ contain the least point, $\wedge F(t)$. Then $F$ admits an increasing selection.

Without any restriction on $F(t)$, Proposition 3.3 is wrong, as was shown in [17, p. 307]. Moreover, its rather strong assumption on $F(t)$ cannot be relaxed even under monotonicity w.r.t. $\geq$.

Example 3.4. Let $X:=\mathbb{N}^{3} \cap[0,5]^{3} \subset \mathbb{R}^{3}, T:=\{(0,0),(0,1),(1,0),(1,1)\} \subset \mathbb{R}^{2}$, and $F: T \rightarrow \mathfrak{B}_{X}$ be defined by $F(0,0):=\{(0,2,2),(2,0,0)\}, F(1,0):=\{(1,4,3),(4,1,3)\}, F(0,1):=$ $\{(3,4,1),(1,3,4)\}$, and $F(1,1):=\{(3,5,3),(5,3,5)\}$. It is easily checked that $F$ is increasing w.r.t. $\geq$; every $F(t)$ is supported both below and above. However, there exists no increasing selection from $F$ : if $r(0,0)=(0,2,2)$, then $r(1,0)=(1,4,3)$ and $r(0,1)=(1,3,4)$; however, there is no $x \in F(1,1)$ such that $x \geq r(1,0)$ and $x \geq r(0,1)$. Supposing $r(0,0)=(2,0,0)$, we obtain a similar contradiction.

The existence of an increasing selection from a multifunction increasing w.r.t. $\geq^{\inf }$ or $\geq^{\text {sup }}$ is ensured if either property, "being finite" and "being a chain," applies to either $T$ or every $F(t)$. However, it may not exist when both $X$ and $T$ are infinite chains, even when $F$ is increasing w.r.t. $\geq$ [16, Example 2.3]. 
Proposition 3.5. If $X$ is a poset, $T$ is a finite chain, and $F: T \rightarrow \mathfrak{B}_{X}$ is increasing w.r.t. $\geq^{\text {inf }}$, then $F$ admits an increasing selection. Moreover, whenever $x^{0} \in F(\max T)$, there is an increasing selection $f$ from $F$ such that $f(\max T)=x^{0}$.

Straightforward recursion along $T$ (downwards).

Proposition 3.6. If $X$ and $T$ are posets, $F: T \rightarrow \mathfrak{B}_{X}$ is increasing w.r.t. $\geq^{\text {inf }}$, and every $F(t)$ is a finite chain, then $F$ admits an increasing selection. Moreover, whenever $T^{+}$defined by (1) is not empty, $T^{*} \subseteq T^{+}$, and $g: T^{*} \rightarrow X$ is a selection from the restriction of $F$ to $T^{*}$, there exists an increasing selection $f$ from $F$ such that $f(t)=g(t)$ for every $t \in T^{*}$.

Proof. The first statement immediately follows from Proposition 3.3. To prove the second, we replace $F$ with $F^{*}$ defined as follows: $F^{*}(t):=\{g(t)\}$ if $t \in T^{*} ; F^{*}(t):=\left\{x \in F(t) \mid \forall t^{\prime} \in T^{*}\left[t^{\prime}>\right.\right.$ $\left.\left.t \Rightarrow g\left(t^{\prime}\right) \geq x\right]\right\}$ otherwise. We have $F^{*}(t) \neq \emptyset$ because $F(t)$ is a finite chain; $F^{*}$ obviously remains increasing w.r.t. $\geq^{\text {inf }}$. Therefore, the first statement applies to $F^{*}$.

Proposition 3.7. If $X$ is a poset, $T$ is a chain, $F: T \rightarrow \mathfrak{B}_{X}$ is increasing w.r.t. $\geq^{\text {inf }}$, and every $F(t)$ is finite, then $F$ admits an increasing selection. Moreover, whenever $\max T$ exists and $x^{0} \in F(\max T)$, there is an increasing selection $f$ from $F$ such that $f(\max T)=x^{0}$.

The proof is deferred to Section 4.4

Proposition 3.8. Let $X$ be a poset, $T$ be a finite poset, $F: T \rightarrow \mathfrak{B}_{X}$ be increasing w.r.t. inf $^{\text {, }}$ each $F(t)$ be a chain, $T^{*} \subseteq T^{+}$[defined by (1)], and $g: T^{*} \rightarrow X$ be a selection from the restriction of $F$ to $T^{*}$. Then there exists an increasing selection $f$ from $F$ such that $f(t)=g(t)$ for every $t \in T^{*}$.

Proof. We start with setting $f(t):=g(t)$ for every $t \in T^{*}$. Since all $t, t^{\prime} \in T^{*}$ are incomparable, there is no problem with monotonicity. Defining $T_{1}:=T \backslash T^{*}$, we denote $S(t):=\left\{t^{\prime} \in T^{*} \mid t^{\prime}>t\right\}$ for every $t \in T_{1}^{+}$. For every $t \in T_{1}^{+}$and $t^{\prime} \in S(t)$, we pick $\xi\left(t, t^{\prime}\right) \in F(t)$ such that $\xi\left(t, t^{\prime}\right) \leq f\left(t^{\prime}\right)$. Then we set $f(t):=\min _{t^{\prime} \in S(t)} \xi\left(t, t^{\prime}\right)$ for every $t \in T_{1}^{+}$; by definition, $f$ is increasing on $T^{*} \cup T_{1}^{+}$. Now we define $T_{2}:=T_{1} \backslash T_{1}^{+}$and continue the procedure until $T$ is exhausted.

Proposition 3.9. If $X$ is a poset, $T$ is a finite chain, $F: T \rightarrow \mathfrak{B}_{X}$ is increasing w.r.t. $\geq, t^{0} \in T$, and $x^{0} \in F\left(t^{0}\right)$, then $F$ admits an increasing selection $f$ such that $f\left(t^{0}\right)=x^{0}$.

Immediately follows from Proposition 3.5 and its dual.

Proposition 3.10. Let $X$ and $T$ be posets, $F: T \rightarrow \mathfrak{B}_{X}$ be increasing w.r.t. $\geq$, and every $F(t)$ be a finite chain; let $T_{0} \in \mathfrak{B}_{T}$ be such that $t \in T_{0}$ whenever $t^{\prime}<t<t^{\prime \prime}$ and $t^{\prime}, t^{\prime \prime} \in T_{0}$, and let $g: T_{0} \rightarrow X$ be an increasing selection from the restriction of $F$ to $T_{0}$. Then there exists an increasing selection $f$ from $F$ such that $f(t)=g(t)$ for every $t \in T_{0}$.

Easily follows from Proposition 3.6 and its dual. 
Proposition 3.11. If $X$ is a poset, $T$ is a chain, $F: T \rightarrow \mathfrak{B}_{X}$ is increasing w.r.t. $\geq$, every $F(t)$ is finite, $t^{0} \in T$, and $x^{0} \in F\left(t^{0}\right)$, then $F$ admits an increasing selection $f$ such that $f\left(t^{0}\right)=x^{0}$.

Immediately follows from Proposition 3.7 and its dual.

Proposition 3.12. Let $X$ be a poset, $T$ be a finite poset, $F: T \rightarrow \mathfrak{B}_{X}$ be increasing w.r.t. $\geq$, and each $F(t)$ be a chain; let $T_{0} \in \mathfrak{B}_{T}$ be such that $t \in T_{0}$ whenever $t^{\prime}<t<t^{\prime \prime}$ and $t^{\prime}, t^{\prime \prime} \in T_{0}$, and let $g: T_{0} \rightarrow X$ be an increasing selection from the restriction of $F$ to $T_{0}$. Then there exists an increasing selection $f$ from $F$ such that $f(t)=g(t)$ for every $t \in T_{0}$.

Easily follows from Proposition 3.8 and its dual.

It may be possible to relax the assumptions in Propositions 3.5- 3.12; however, one could hardly go very far. For instance, finiteness cannot be replaced with the absence of infinite chains.

Example 3.13. Let $X:=\{(x-1,-2-x)\}_{x \in \mathbb{R},-1<x<0} \cup\{(-1,1),(1,-1)\} \cup\{(x+1,2-x)\}_{x \in \mathbb{R}, 0<x<1}$ $\subset \mathbb{R}^{2}$ and $T:=[-1,1]$, both with the natural order. $T$ is a chain; $X$ contains no infinite chain. Let $F: T \rightarrow \mathfrak{B}_{X}$ be defined by: $F(-1):=\{(x-1,-2-x)\}_{x \in \mathbb{R},-1<x<0} ; F(t):=$ $\{(x-1,-2-x)\}_{x \in \mathbb{R}, t<x<0} \cup\{(1,-1)\}$ whenever $-1<t<0 ; F(0):=\{(-1,1),(1,-1)\} ; F(t):=$ $\{(x+1,2-x)\}_{x \in \mathbb{R}, t<x<1} \cup\{(-1,1)\}$ whenever $0<t<1 ; F(1):=\{(x+1,2-x)\}_{x \in \mathbb{R}, 0<x<1}$. $F$ is increasing w.r.t. $\geq$; however, if we assume that $f: T \rightarrow X$ is an increasing selection from $F$, we immediately obtain $f(t)=(1,-1)$ for some $t<0$ and $f(t)=(-1,1)$ for some $t>0$.

Example 3.14. Let $X:=\mathbb{Z}$ with the natural order and $T:=\{\top, \perp\} \cup\left\{t_{h}^{k}\right\}_{k \in \mathbb{N}, 0 \leq h<2 k}$ with this order: $\top>t_{h}^{k}>\perp$ for each $t_{h}^{k} \in T \backslash\{\top, \perp\}, t_{h^{\prime}}^{k}>t_{h}^{k}$ whenever $h^{\prime}>h$, and $t_{h^{\prime}}^{k^{\prime}}$ is incomparable with $t_{h}^{k}$ whenever $k^{\prime} \neq k$ (actually, $T$ can be imbedded into $\mathbb{R}^{2}$, but that would not make things clearer). $X$ is a chain, $T$ contains no infinite chain. Let $F: T \rightarrow \mathfrak{B}_{X}$ be defined by: $F(T):=F(\perp):=\mathbb{Z}$, $F\left(t_{h}^{k}\right):=2 \mathbb{Z}$ whenever $h \in 2 \mathbb{Z}$, and $F\left(t_{h}^{k}\right):=2 \mathbb{Z}+1$ whenever $h \notin 2 \mathbb{Z}$. $F$ is increasing w.r.t. $\geq$, but admits no increasing selection for the same reason as in [16, Example 2.3].

By Proposition 3.2, Propositions 3.3 and $3.5-3.12$ apply to monotonicity w.r.t. (3a) - (3c). Concerning the latter relation, we have a much stronger result, which appears to be new.

Theorem 3. If $X$ and $T$ are posets, $F: T \rightarrow \mathfrak{B}_{X}$ is increasing w.r.t. $\geqslant, t^{0} \in T$, and $x^{0} \in F\left(t^{0}\right)$, then $F$ admits an increasing selection $f$ such that $f\left(t^{0}\right)=x^{0}$.

Proof. Invoking Zermelo's Theorem, we assume $X$ well ordered with an order $\gg$ (generally, having nothing to do with the basic order on $X$ ). Moreover, we assume that $x^{0}=\min X$ (in the sense of $\gg)$.

Now we define $f(t):=\min F(t)$ (w.r.t. $\gg$ ); it exists and is unique for every $t \in T . f$ is a selection from $F$ by definition; moreover, $f(t)=x^{0}$ whenever $x^{0} \in F(t)$. Let $t^{\prime}>t, y=f\left(t^{\prime}\right)$ and $x=f(t)$. Since $F$ is increasing w.r.t. $\geqslant$, we have $y>x$ unless $y \in F\left(t^{\prime}\right) \cap F(t) \ni x$. In the latter case, we must have $y=x=\min \left(F\left(t^{\prime}\right) \cap F(t)\right)$. Thus, $f$ is increasing. 
Relation (3d) has no analog among relations (4). If every $F(t)$ is a chain, then Proposition 3.1 allows us to invoke Theorem 1 if every $F(t)$ is complete, or Proposition 2.4 if $T$ is finite. If $T$ is a chain, then some analogs of Propositions 3.5 and 3.7 can be obtained.

Let $X$ be a poset, $T$ be a chain, and $F: T \rightarrow \mathfrak{B}_{X}$ be increasing w.r.t. $\geqslant^{\text {w }}$. For any $\bar{t} \in T$ and $\bar{x} \in F(\bar{t})$ we define $F^{\bar{t}, \bar{x}}: T \rightarrow 2^{X}$ by:

$$
F^{\bar{t}, \bar{x}}(t) \rightleftharpoons \begin{cases}\{x \in F(t) \mid x \geq \bar{x}\}, & t>\bar{t} \\ \{\bar{x}\}, & t=\bar{t} \\ \{x \in F(t) \mid x \leq \bar{x}\}, & t<\bar{t}\end{cases}
$$

Lemma 3.15. For every $t \neq \bar{t}$, either $F^{\bar{t}, \bar{x}}(t) \neq \emptyset$ or $F(t) \subset F(\bar{t})$.

Lemma 3.16. The mapping $F^{\bar{t}, \bar{x}}: T \rightarrow 2^{X}$ is increasing w.r.t. $\geqslant^{\mathrm{w}}$.

Both proofs are straightforward.

Proposition 3.17. If $X$ is a poset, $T$ is a finite chain, and $F: T \rightarrow \mathfrak{B}_{X}$ is increasing w.r.t. $\geqslant^{w}$, then $F$ admits an increasing selection.

Proof. First, we pick $\bar{t} \in T$ such that $F(\bar{t})$ is minimal w.r.t. inclusion, and pick $\bar{x} \in F(\bar{t})$ arbitrarily. By Lemmas 3.15 and $3.16, F^{\bar{t}, \bar{x}}$ is a multifunction increasing w.r.t. $\geqslant^{\mathrm{w}}$. Then we apply the same construction to the restriction of $F^{\bar{t}, \bar{x}}$ to $T \backslash\{\bar{t}\}$, and repeat until $T$ is exhausted.

Proposition 3.18. If $X$ is a poset, $T$ is a chain, $F: T \rightarrow \mathfrak{B}_{X}$ is increasing w.r.t. $\geqslant^{\mathrm{w}}$, and every $F(t)$ is finite, then $F$ admits an increasing selection.

The proof is deferred to Section 4.5 .

Example 3.19. Let $X:=T:=\{(0,0),(0,1),(1,0)\} \subset \mathbb{R}^{2}$, and $F: T \rightarrow \mathfrak{B}_{X}$ be defined by $F(0,0):=\{(0,1),(1,0)\}, F(1,0):=\{(1,0)\}$, and $F(0,1):=\{(0,1)\}$. It is easily checked that $F$ is increasing w.r.t. $\geqslant^{\text {Up }}$. Both $X$ and $T$ are finite, but $T$ and $F(0,0)$ are not chains. Clearly, there exists no increasing selection from $F$.

Example 3.20. Let $X:=\{(k,-k)\}_{k \in \mathbb{N}} \subset \mathbb{R}^{2}$ and $T:=\mathbb{N}$, both with the natural orders. $T$ is a chain; $X$ contains no infinite chain. Let $F: T \rightarrow \mathfrak{B}_{X}$ be defined by $F(m):=\{(k,-k)\}_{k \in \mathbb{N}, k \geq m}$. $F$ is increasing w.r.t. $\geqslant^{\mathrm{Up}}$; however, there is no increasing selection from $F$.

Finally, Example 2.3 shows that the existence of an increasing selection with a given value at a given point cannot be guaranteed even when both $X$ and $T$ are finite chains.

\section{Proofs}

\subsection{Proof of Theorem 1}

We define $\mathfrak{F}$ as the set of mappings $G: T \rightarrow \mathfrak{B}_{X}$ such that:

$$
\forall t \in T\left[F^{-}(t) \subseteq G(t) \subseteq F(t)\right]
$$




$$
\begin{aligned}
& \forall t \in T \forall y, x \in F(t)[[y>x \& y \in G(t)] \Rightarrow x \in G(t)] ; \\
& \forall t \in T[G(t) \text { is chain-subcomplete upwards in } F(t)] ; \\
& G \text { is increasing w.r.t. } \geq^{\mathrm{wV}} .
\end{aligned}
$$

Clearly, $F \in \mathfrak{F} \neq \emptyset$. We define $\bar{G}: T \rightarrow \mathfrak{B}_{X}$ by

$$
\bar{G}(t):=\bigcap_{G \in \mathfrak{F}} G(t)
$$

for every $t \in T$.

Lemma 4.1.1. $\bar{G} \in \mathfrak{F}$.

Proof. Conditions (7a), (7b), and (7c) are satisfied trivially; only $(\overline{7 d})$ deserves some attention. Let $t^{\prime}>t, y \in \bar{G}\left(t^{\prime}\right)$, and $x \in \bar{G}(t)$; then $y \in G\left(t^{\prime}\right)$ and $x \in G(t)$ for every $G \in \mathfrak{F}$. If $y \wedge x \in F(t)$, then $y \wedge x \in G(t)$ for every $G \in \mathfrak{F}$ by $(\overline{7 b})$ for $G$, hence $y \wedge x \in \bar{G}(t)$. Otherwise, $y \vee x \in G\left(t^{\prime}\right)$ for every $G \in \mathfrak{F}$ by $(\overline{7 d})$ for $G$, hence $y \vee x \in \bar{G}\left(t^{\prime}\right)$. Since $y$ and $x$ were arbitrary, $\bar{G}\left(t^{\prime}\right) \geq^{\mathrm{wV}} \bar{G}(t)$, hence $\bar{G} \in \mathfrak{F}$ indeed.

For every $G \in \mathfrak{F}$ and $t \in T$, we have $G^{+}(t) \neq \emptyset$ since $G(t)$ is chain-complete upwards.

Lemma 4.1.2. If $G \in \mathfrak{F}, t^{\prime}>t, y \in G^{+}\left(t^{\prime}\right)$ and $x \in F^{-}(t)$, then $y \geq x$.

Proof. Otherwise, we would have $y \vee x>y$ and $y \wedge x<x$; therefore, $y \vee x \notin G\left(t^{\prime}\right)$ and $y \wedge x \notin F(t)$, contradicting our assumption $G\left(t^{\prime}\right) \geq^{\mathrm{wV}} G(t) \subseteq F(t)$.

For any $G \in \mathfrak{F}$, we define its transformation $G^{*}: T \rightarrow \mathfrak{B}_{X}$ by

$$
G^{*}(t):=\left\{x \in G(t) \mid \forall t^{\prime}>t \forall y \in G^{+}\left(t^{\prime}\right)[y \geq x]\right\} .
$$

Lemma 4.1.3. For every $G \in \mathfrak{F}$, there holds $G^{*} \in \mathfrak{F}$.

Proof. Condition (7a) immediately follows from Lemma 4.1 .2 and $(9) ;(7 \mathrm{~b})$ is obvious. To check (7c), we fix $t \in T$ and consider a chain $Z \subseteq G^{*}(t)$; we have to show that $\sup Z \in G^{*}(t)$ ("sup" here means the least upper bound in $F(t)$; it may depend on $t$ ). Suppose $x:=\sup Z \notin G^{*}(t)$, i.e., there are $t^{\prime}>t$ and $y \in G^{+}\left(t^{\prime}\right)$ such that $y \nsupseteq x$; then we have $x>y \wedge x$. On the other hand, $y \wedge x \in G(t) \subseteq F(t)$ because $G\left(t^{\prime}\right) \geq^{\mathrm{wV}} G(t)$ and $y \in G^{+}\left(t^{\prime}\right)$; for every $z \in Z$, we have $x \geq z$ by the definition of $x$ and $y \geq z$ because $z \in G^{*}(t)$, hence $y \wedge x \geq z$ as well. Clearly, we have a contradiction with the definition of $x$.

Finally, let us check (7d); let $t^{\prime}>t, y \in G^{*}\left(t^{\prime}\right)$, and $x \in G^{*}(t)$. If $y \wedge x \in G(t)$, then $y \wedge x \in G^{*}(t)$ by (7b) for $G^{*}$. Otherwise, $y \vee x \in G\left(t^{\prime}\right)$. For every $t^{\prime \prime}>t^{\prime}$ and $z \in G^{+}\left(t^{\prime \prime}\right)$, we have $z \geq x$ because $x \in G^{*}(t)$, and $z \geq y$ because $y \in G^{*}\left(t^{\prime}\right)$. Therefore, $z \geq y \vee x$; since $t^{\prime \prime}$ and $z$ were arbitrary, $y \vee x \in G^{*}\left(t^{\prime}\right)$. 
Lemma 4.1.4. $\bar{G}=\bar{G}^{*}$.

Immediately follows from (8), (9), and Lemma 4.1.3.

Finally, let $f: T \rightarrow X$ be an arbitrary selection from $\bar{G}^{+}$. Lemma 4.1 .4 and (9) immediately imply that $f$ is increasing.

\subsection{Proof of Proposition 2.4}

For every $t \in T$, we denote $T^{\downarrow}(t):=\left\{t^{\prime} \in T \mid t^{\prime}<t\right\}$; for every $t \in T$ and $x^{*} \in X, F^{\downarrow}\left(t ; x^{*}\right):=$ $\left\{x \in F(t) \mid x \leq x^{*}\right\}$.

Lemma 4.2.1. For every $t^{*} \in T^{+}\left[\right.$the latter defined by (1)], there exists $x^{*} \in F\left(t^{*}\right)$ such that $F^{\downarrow}\left(t ; x^{*}\right) \neq \emptyset$ for every $t \in T^{\downarrow}\left(t^{*}\right)$.

Proof. For every $x \in F\left(t^{*}\right)$, we denote $S(x):=\left\{t \in T^{\downarrow}\left(t^{*}\right) \mid F^{\downarrow}(t ; x) \neq \emptyset\right\}$. Then we pick $x^{0} \in$ $F\left(t^{*}\right)$ arbitrarily and define a sequence $x^{0}, x^{1}, \ldots$ by recursion. Let $x^{k} \in F\left(t^{*}\right)$ have been defined. If $S\left(x^{k}\right)=T^{\downarrow}\left(t^{*}\right)$, we take $x^{k}$ as $x^{*}$ and finish the process; otherwise, we pick $t \in T^{\downarrow}\left(t^{*}\right) \backslash S\left(x^{k}\right)$ arbitrarily. We have $F\left(t^{*}\right) \geq^{\mathrm{wV}} F(t)$; picking $z \in F(t)$ arbitrarily, we apply (2d) with $y=x^{k}$. Since $F^{\downarrow}\left(t ; x^{k}\right)=\emptyset$, we have $z \wedge x^{k} \notin F(t)$, hence $z \vee x^{k} \in F\left(t^{*}\right)$. Defining $x^{k+1}:=z \vee x^{k}>x^{k}$, we obtain $S\left(x^{k}\right) \subset S\left(x^{k+1}\right)$. Since $T$ is finite, the sequence must stabilize at some stage, i.e., reach the situation $S\left(x^{k}\right)=T^{\downarrow}\left(t^{*}\right)$.

Now the proposition is proven with straightforward induction in \#T. Picking $t^{*} \in T^{+}$arbitrarily and $x^{*}$ as in Lemma 4.2.1, we define $\bar{F}: T \backslash\left\{t^{*}\right\} \rightarrow X$ by $\bar{F}(t):=F^{\downarrow}\left(t ; x^{*}\right)$ for $t \in T^{\downarrow}\left(t^{*}\right)$ and $\bar{F}(t):=F(t)$ otherwise. Obviously, $\bar{F}(t)$ is increasing w.r.t. $\geq^{\mathrm{wV}}$, hence it admits an increasing selection $f$ by the induction hypothesis. Adding $f\left(t^{*}\right):=x^{*}$, we obtain an increasing selection from $F$ on $T$.

\subsection{Proof of Theorem 2}

The argument is similar to the proof of Theorem 3 , but a bit more complicated. Let $X \subseteq$ $\prod_{m \in M} C_{m}$, where each $C_{m}$ is a chain. We assume $M$ totally ordered, say, $M=\{0,1, \ldots, \bar{m}\}$; then, invoking Zermelo's Theorem, we assume each $C_{m}$ well ordered with an order $\gg_{m}$. Moreover, we assume that $x_{m}^{0}=\min C_{m}$ (in the sense of $\gg_{m}$ ) for each $m \in M$. Given $y \neq x$, we define $D(y, x):=\left\{m \in M \mid y_{m} \neq x_{m}\right\}, d:=\min D(y, x)$, and $y \gg x \rightleftharpoons y_{d} \gg_{d} x_{d}$.

Lemma 4.3.1. $X$ is well ordered by $\gg$.

Proof. Being a lexicographic combination, $\gg$ is clearly an order. For every $Y \in \mathfrak{B}_{X}$, we define $c_{1}:=\min \left\{y_{1} \mid y \in Y\right\}, c_{2}:=\min \left\{y_{2} \mid y \in Y \& y_{1}=c_{1}\right\}$, etc. (the minima are w.r.t. $\gg_{1}, \gg_{2}$, etc.); then $\left(c_{1}, c_{2}, \ldots, c_{\bar{m}}\right)$ is the minimum of $Y$ w.r.t. $\gg$.

Lemma 4.3.2. Let $x, y \in X$ and $y \nsupseteq x$. Then $x \gg y \wedge x \Longleftrightarrow y \vee x \gg y$. 
Proof. Denoting $D^{-}:=\left\{m \in M \mid y_{m}<x_{m}\right\}$, we immediately see that $D^{-}=D(y, y \vee x)=$ $D(x, y \wedge x)$; let $d:=\min D^{-}$. If $x_{d} \gg_{d} y_{d}$, then $x \gg y \wedge x$ and $y \vee x \gg y$. If $y_{d} \gg_{d} x_{d}$, then $y \gg y \vee x$ and $y \wedge x \gg x$.

Remark. We might say that $\gg$ is "quasimodular."

Now we define $f(t):=\min F(t)$ (w.r.t. $\gg$ ); it exists and is unique. $f$ is a selection from $F$ by definition; moreover, $f(t)=x^{0}$ whenever $x^{0} \in F(t)$. Let us show that $f$ is increasing. Let $t^{\prime}>t$, $y=f\left(t^{\prime}\right)$ and $x=f(t)$; since $F$ is increasing w.r.t. $\geq^{\mathrm{Vt}}$, we have $y \wedge x \in F(t)$ and $y \vee x \in F\left(t^{\prime}\right)$. If $y \nsupseteq x$, then $x \neq y \wedge x$ and $y \neq y \vee x$, hence $y \wedge x \gg x$ and $y \vee x \gg y$ by the definition of $f$. Thus, we have a contradiction with Lemma 4.3.2.

\subsection{Proof of Proposition 3.7}

Lemma 4.4.1. Let $T^{\prime} \subseteq T, G: T^{\prime} \rightarrow \mathfrak{B}_{X}$ be increasing w.r.t. $\geq^{\inf }$, $\max T^{\prime}$ exist, and $y \in$ $G\left(\max T^{\prime}\right)$. For every $t \in T^{\prime}$, we define

$$
G_{\leftarrow}^{y}(t):= \begin{cases}\{y\} & t=\max T^{\prime} \\ \{x \in G(t) \mid y \geq x\}, & t<\max T^{\prime} .\end{cases}
$$

Then $G_{\leftarrow}^{y}: T^{\prime} \rightarrow \mathfrak{B}_{X}$ is increasing w.r.t. $\geq^{\inf }$.

The straightforward proof is omitted.

We define $\mathfrak{F}$ as the set of mappings $G: T \rightarrow \mathfrak{B}_{X}$ such that:

$$
\begin{gathered}
\forall t \in T[G(t) \subseteq F(t)] ; \\
G \text { is increasing w.r.t. } \geq^{\text {inf }} .
\end{gathered}
$$

Clearly, $F \in \mathfrak{F} \neq \emptyset$. We introduce a partial order on $\mathfrak{F}$ by $G^{\prime} \geq G \rightleftharpoons \forall t \in T\left[G^{\prime}(t) \subseteq G(t)\right]$.

Lemma 4.4.2. There exists a maximal $\bar{G} \in \mathfrak{F}$, i.e., $\forall G \in \mathfrak{F}[G \geq \bar{G} \Rightarrow G=\bar{G}]$.

Proof. Let $\left\{G_{\alpha}\right\}_{\alpha \in \mathrm{A}}$ be a chain in $\mathfrak{F}$. For every $t \in T$, we define $G^{+}(t):=\bigcap_{\alpha \in \mathrm{A}} G_{\alpha}(t)$. Since $F(t)$ itself is finite, $G^{+}(t)=\emptyset$ would imply $G_{\alpha}(t)=\emptyset$ for some $\alpha \in$ A. Clearly, $G^{+} \in \mathfrak{F}$. A reference to Zorn's Lemma finishes the proof.

For any $G \in \mathfrak{F}$, we define its transformation by

$$
G^{*}(t):=\left\{x \in G(t) \mid \forall t^{\prime}>t \exists y \in G\left(t^{\prime}\right)[y \geq x]\right\} .
$$

Lemma 4.4.3. For every $G \in \mathfrak{F}$ and $t \in T$, there holds $G^{*}(t) \neq \emptyset$. 
Proof. Supposing the contrary, we may pick $\tau(x)>t$ for every $x \in G(t)$ such that the inequality $y \geq x$ does not hold for any $y \in G(\tau(x))$. Since $G(t)$ is finite, it can be numbered, $G(t)=$ $\left\{x^{1}, \ldots, x^{m}\right\}$, such that $\tau\left(x^{k}\right) \geq \tau\left(x^{k+1}\right)$ for each $k=1, \ldots, m-1$. Then we pick $y^{1} \in \tau\left(x^{1}\right)$ arbitrarily, and recursively construct a sequence $y^{1}, \ldots, y^{m}$ such that $y^{k} \in G\left(\tau\left(x^{k}\right)\right)$ and $y^{k} \geq y^{k+1}$ for each $k$; this is possible because $G\left(\tau\left(x^{k}\right)\right) \geq^{\inf } G\left(\tau\left(x^{k+1}\right)\right)$. Since $G\left(\tau\left(x^{m}\right)\right) \geq^{\inf } G(t)$, there must be $x^{k} \in G(t)$ such that $y^{m} \geq x^{k}$, but then $y^{k} \geq x^{k}$, which contradicts the definition of $\tau$.

Lemma 4.4.4. For every $G \in \mathfrak{F}$, there holds $G^{*} \in \mathfrak{F}$.

Proof. By Lemma 4.4.3, $G^{*}$ maps $T$ to $\mathfrak{B}_{X}$; (11a) is obvious. To check (11b), let $t^{\prime \prime}>t^{\prime}$ and $y \in G^{*}\left(t^{\prime \prime}\right) \subseteq G\left(t^{\prime \prime}\right)$. We define $T^{\prime}:=\left\{t \in T \mid t \leq t^{\prime \prime}\right\}$; clearly, $t^{\prime \prime}=\max T^{\prime}$, hence Lemma 4.4.1 is applicable to the restriction of $G^{*}$ to $T^{\prime}$, giving us an appropriate $G^{\prime}$. Now we can reproduce the definition of $\mathfrak{F}\left(\mathfrak{F}^{\prime}\right.$, so to speak) and of the transformation, and then apply Lemma 4.4.3 with $t=t^{\prime}$, obtaining $x \in G^{*}\left(t^{\prime}\right) \subseteq G^{*}\left(t^{\prime}\right)$. By (10), $y \geq x$.

Lemma 4.4.5. If $\bar{G} \in \mathfrak{F}$ is maximal in $\mathfrak{F}$, then $\bar{G}$ is increasing w.r.t. $\geq$.

Proof. By (12) and Lemma 4.4.4, $\bar{G}^{*}=\bar{G}$. By the same (12), this means that $\bar{G}$ is increasing w.r.t. $\geq$.

Lemma 4.4.6. If $\bar{G} \in \mathfrak{F}$ is maximal in $\mathfrak{F}$, then $\# \bar{G}(t)=1$ for every $t \in T$.

Proof. Fixing $\bar{t} \in T$ and $y \in \bar{G}(\bar{t})$ arbitrarily, we define $T^{\prime}:=\{t \in T \mid t \leq \bar{t}\}$ and $T^{\prime \prime}:=$ $\{t \in T \mid t \geq \bar{t}\}$; clearly, $\bar{t}=\max T^{\prime}=\min T^{\prime \prime}$. We define $G^{\prime}:=G_{\leftarrow}^{y}: T^{\prime} \rightarrow \mathfrak{B}_{X}$ by (10), and $G^{\prime \prime}:=G_{\rightarrow}^{y}: T^{\prime \prime} \rightarrow \mathfrak{B}_{X}$ dually; by Lemma 4.4 .1 and its dual version, $G^{\prime}$ is increasing w.r.t. ${ }^{\text {inf }}$, whereas $G^{\prime \prime}$ is increasing w.r.t. $\geq^{\text {sup }}$. By Lemma 4.4 .5 applied to $G^{\prime}$, and its dual version applied to $G^{\prime \prime}$, there are multifunctions $\bar{G}^{\prime}: T^{\prime} \rightarrow \mathfrak{B}_{X}$ and $\bar{G}^{\prime \prime}: T^{\prime \prime} \rightarrow \mathfrak{B}_{X}$, both increasing w.r.t. $\geq$, such that $\bar{G}^{\prime}(t) \subseteq \bar{G}(t)$ for every $t \in T^{\prime}, \bar{G}^{\prime \prime}(t) \subseteq \bar{G}(t)$ for every $t \in T^{\prime \prime}$, and $\bar{G}^{\prime}(\bar{t})=\bar{G}^{\prime \prime}(\bar{t})=\{y\}$. Denoting $G$ their combination, i.e., $G(t):=\bar{G}^{\prime}(t)$ for $t \leq \bar{t}$ and $G(t):=\bar{G}^{\prime \prime}(t)$ for $t \geq \bar{t}$, we see that $G \in \mathfrak{F}$ and $G \geq \bar{G}$. Since $\bar{G}$ is maximal, $G=\bar{G}$, hence $\bar{G}(\bar{t})=G(\bar{t})=\{y\}$.

Now the first statement of Proposition 3.7 immediately follows from Lemma 4.4.6. To prove the second statement, we first apply Lemma 4.4 .1 to $F$ itself as $G, T$ as $T^{\prime}$, and $x^{0} \in \max T$ as $y$; then we replace $F$ with $F^{\prime}$ defined by (10) in the definition of $\mathfrak{F}$ and all the following constructions.

\subsection{Proof of Proposition 3.18}

We define $\mathfrak{F}$ as the set of mappings $G: T \rightarrow \mathfrak{B}_{X}$ such that:

$$
\begin{gathered}
\forall t \in T[G(t) \subseteq F(t)] ; \\
G \text { is increasing w.r.t. } \geqslant^{\mathrm{w}} .
\end{gathered}
$$

Clearly, $F \in \mathfrak{F} \neq \emptyset$. We introduce a partial order on $\mathfrak{F}$ by $G^{\prime} \geq G \rightleftharpoons \forall t \in T\left[G^{\prime}(t) \subseteq G(t)\right]$. 
Lemma 4.5.1. There exists a maximal $\bar{G} \in \mathfrak{F}$, i.e., $\forall G \in \mathfrak{F}[G \geq \bar{G} \Rightarrow G=\bar{G}]$.

The proof is virtually identical with that of Lemma 4.4.2.

For the rest of the proof, we fix an arbitrary maximal $\bar{G} \in \mathfrak{F}$.

Lemma 4.5.2. Whenever $\bar{G}(\bar{t})$ is minimal w.r.t. inclusion among $\bar{G}(t)(t \in T)$, there holds $\bar{G}(\bar{t})=\{\bar{x}\}$. Besides, $x \geq \bar{x}$ whenever $x \in \bar{G}(t)$ and $t>\bar{t}$, while $x \leq \bar{x}$ whenever $x \in \bar{G}(t)$ and $t<\bar{t}$.

Proof. Let us pick $\bar{x} \in \bar{G}(\bar{t})$ arbitrarily and consider $\bar{G}^{\bar{t}}, \bar{x}$ defined by (6). By Lemmas 3.15 and 3.16 , $\bar{G}^{\bar{t}, \bar{x}} \in \mathfrak{F}$, hence $\bar{G}^{\bar{t}, \bar{x}}=\bar{G}$, hence $\bar{G}(\bar{t})=\{\bar{x}\}$. The second statement immediately follows from (6) and $\bar{G}^{\bar{t}}, \bar{x}=\bar{G}$.

By induction in $m \in \mathbb{N}$, we prove the following statement: Whenever $\# \bar{G}(\bar{t}) \leq m$, there holds $\bar{G}(\bar{t})=\{f(\bar{t})\}$ such that $x \geq f(\bar{t})$ for $x \in \bar{G}(t)$ and $t>\bar{t}$, and $x \leq f(\bar{t})$ for $x \in \bar{G}(t)$ and $t<\bar{t}$. When $m=1$, Lemma 4.5 .2 applies.

Suppose that our statement holds for $m$ and let $\# \bar{G}(\bar{t})=m+1$. We pick $\bar{x} \in \bar{G}(\bar{t})$ arbitrarily and consider $\bar{G}^{\bar{t}, \bar{x}}$ defined by (6); it is increasing w.r.t. $\geqslant^{\text {w }}$ by Lemma 3.16 . Let $t>\bar{t}$; by the induction hypothesis, we have either $\# \bar{G}(t) \geq m+1$ or $\# \bar{G}(t)=1$. In the first case, we apply Lemma 3.15 to $\bar{t}$ and $t$; in the second, Lemma 4.5.2 to $t$ and $\bar{t}$. The case of $t<\bar{t}$ is treated dually. Again, $\bar{G}^{\bar{t}, \bar{x}} \in \mathfrak{F}$, hence $\bar{G}^{\bar{t}, \bar{x}}=\bar{G}$. Setting $f(\bar{t})=\bar{x}$, we see that our statement holds for $m+1$ as well.

Once the induction process is finished, we have $\bar{G}(t)=\{f(t)\}$ for every $t \in T$, and $f$ is an increasing selection from $F$.

\section{Concluding remarks}

5.1. To my knowledge, there is no example of an ascending multifunction without an increasing selection. However, the current proof of Theorem 2 is useless even if $X$ is a sublattice of the Cartesian product of an infinite number of chains, to say nothing of a non-distributive lattice. Vladimir Danilov (personal communication, 2007) proved the theorem for an arbitrary lattice $X$, but a countable poset $T$.

5.2. The strategy sets in game models are often sublattices of $\mathbb{R}^{m}$, so Theorem 2 is sufficient to prove the existence of a Nash equilibrium under strategic complementarity; only the existence of the best responses is needed, not their (sub)completeness.

5.3. An anonymous referee suggested another reason to be interested in increasing selections: monotone comparative statics of optima (or equilibria). There are conditions on the objective function that ensure the monotonicity of optima w.r.t. relations (2) or their analogs [10, 13, 15, 9]. The possibility to derive the existence of monotone selections from such monotonicity adds more "flesh" to those results. On the other hand, as the same referee noted, our main results establish 
just the existence of the selections without saying anything specific about them. Unfortunately, even in the case of, more or less, constructive proofs (e.g., Theorem 1), it is not at all clear which choices from each particular $F(t)$ are compatible with monotonicity and which are not.

5.4. It is interesting to note that both relations (2) and (3) form isomorphic lattices (with the logical implication as order), which are not sublattices of the lattice of all binary relations on $\mathfrak{B}_{X}$. Another isomorphic lattice can be obtained by supplementing (4) with this relation

$$
Y \geq^{\mathrm{w}} Z \rightleftharpoons \exists y \in Y \exists z \in Z[y \geq z],
$$

which was considered in [10]. Then Proposition 3.2 becomes valid w.r.t. all the four relations (3). However, this kind of monotonicity does not ensure the existence of an increasing selection even when both $X$ and $T$ are finite chains.

5.5. All relations (2), (3) and (5), unlike (4), hold vacuously if $Y$ or $Z$ is empty. On the other hand, if $(3 \mathrm{c})$ or $(5)$ is extended to $2^{X}$, its transitivity is lost. (Besides, correspondences with empty values cannot admit increasing selections.)

\section{Acknowledgments}

Financial support from the Russian Foundation for Basic Research (projects 11-07-00162 and 1101-12136) and the Spanish Ministry of Education and Innovation (project ECO 2010-19596) is acknowledged. I have benefitted from fruitful contacts with Vladimir Danilov, Francisco Marhuenda, Paul Milgrom, Hervé Moulin, Kevin Reffett, Alexei Savvateev, and Satoru Takahashi. Helpful suggestions by an anonymous referee are also appreciated.

\section{References}

[1] Abian, S., Brown, A.B.: A theorem on partially ordered sets with applications to fixed point theorems. Canad. J. Math. 13, 78-83 (1961) 2

[2] Dubey, P., Haimanko, O., Zapechelnyuk, A.: Strategic complements and substitutes, and potential games. Games Econ. Behav. 54, 77-94 (2006) 2

[3] Höft, H.: Order preserving selections for multifunctions. In: Czermak, J. et al. (eds.) Contributions to General Algebra 5, pp. 189-195. Hölder-Pichler-Tempsky, Wien (1987) 2

[4] Jensen, M.K.: Aggregative games and best-reply potentials. Econ. Theory 43, 45-66 (2010) 2

[5] Kukushkin, N.S.: A fixed-point theorem for decreasing mappings. Econ. Let. 46, 23-26 (1994) 2 
[6] Kukushkin, N.S.: Potentials for binary relations and systems of monotonic reactions. Doklady Akademii Nauk 373, 23-25 (2000) [in Russian; an English translation in Doklady Math. 62, $16-18(2000)] 2$

[7] Kukushkin, N.S.: Best response dynamics in finite games with additive aggregation. Games Econ. Behav. 48, 94-110 (2004) 2

[8] Kukushkin, N.S.: Monotonicity-based conditions for the acyclicity of Cournot tatonnement. Doklady Akademii Nauk 413, 457-460 (2007) [in Russian; an English translation in Doklady Math. 75, 273-276 (2007)] 2

[9] Kukushkin, N.S.: Monotone comparative statics: changes in preferences versus changes in the feasible set. Econ. Theory, to appear DOI: 10.1007/s00199-011-0677-8 (2011) 13

[10] LiCalzi, M., Veinott, A.F., Jr.: Subextremal functions and lattice programming. Available via http://ideas.repec.org/p/wpa/wuwpge/0509001.html Cited 17 March 2012 (1992) 5, 13. 14

[11] Markowsky, G.: Chain-complete posets and directed sets with applications. Algebra Universalis 6, 53-68 (1976) 2

[12] Milgrom, P., Roberts, J.: Rationalizability, learning, and equilibrium in games with strategic complementarities. Econometrica 58, 1255-1277 (1990) 2

[13] Milgrom, P., Shannon, C.: Monotone comparative statics. Econometrica 62, 157-180 (1994) 13

[14] Novshek, W.: On the existence of Cournot equilibrium. Rev. Econ. Stud. 52, 85-98 (1985) 2

[15] Quah, J.: The comparative statics of constrained optimization problems. Econometrica 75, 401-431 (2007) 13

[16] Roddy, M.S., Schröder, B.S.W.: Isotone relations revisited. Discr. Math. 290, 229-248 (2005) $2,5,7$

[17] Smithson, R.E.: Fixed points of order preserving multifunctions. Proc. Amer. Math. Soc. 28, 304-310 (1971) 2, 5

[18] Tarski, A.: A lattice-theoretical fixpoint theorem and its applications. Pacific J. Math. 5, 285-309 (1955) 2

[19] Topkis, D.M.: Equilibrium points in nonzero-sum n-person submodular games. SIAM J. Control Optim. 17, 773-787 (1979) 2

[20] Veinott, A.F., Jr.: Lattice Programming. Unpublished lectures (1989) 2, 3, 4

[21] Vives, X.: Nash equilibrium with strategic complementarities. J. Math. Econ. 19, 305-321 (1990) 2 